\title{
Expiratory Rib Cage Compression in Mechanically Ventilated Subjects: A Randomized Crossover Trial
}

\author{
Fernando S Guimarães PT PhD, Agnaldo J Lopes MD PhD, Sandra S Constantino PT, \\ Juan C Lima PT, Paulo Canuto PT, and Sara Lucia Silveira de Menezes PT PhD
}

\begin{abstract}
BACKGROUND: Expiratory rib cage compression (ERCC) has been empirically used by physiotherapists with the rationale of improving expiratory flows and therefore the airway clearance in mechanically ventilated patients. This study evaluates the acute mechanical effects and sputum clearance of an ERCC protocol in ventilated patients with pulmonary infection. METHODS: In a randomized crossover study, sputum production and respiratory mechanics were evaluated in 20 mechanically ventilated subjects submitted to 2 interventions. ERCC intervention consisted of a series of manual bilateral ERCCs, followed by a hyperinflation maneuver. Control intervention (CTRL) followed the same sequence, but instead of the compressive maneuver, the subjects were kept on normal ventilation. Static $\left(\mathbf{C}_{\mathrm{st}}\right)$ and effective $\left(\mathbf{C}_{\text {eff }}\right)$ compliance and total $\left(\mathbf{R}_{\text {tot }}\right)$ and initial $\left(R_{\text {init }}\right)$ resistance of the respiratory system were measured pre-ERCC (baseline), post-ERCC or CTRL (POST1), and post-hyperinflation (POST2). Peak expiratory flow (PEF) and the flow at 30\% of the expiratory tidal volume (flow $30 \% \mathrm{~V}_{\mathrm{T}}$ ) were measured during the maneuver. RESULTS: ERCC cleared 34.4\% more secretions than CTRL (1 [0.5-1.95] vs 2 [1-3.25], $P<.01)$. Respiratory mechanics showed no differences between control and experimental intervention in POST1 for $\mathrm{C}_{\mathrm{st}}$, $\mathrm{C}_{\text {eff }}, \mathbf{R}_{\text {tot }}$, and $\mathbf{R}_{\text {init }^{\circ}}$ In POST2, ERCC promoted an increase in $\mathrm{C}_{\mathrm{st}}(38.7 \pm 10.3 \mathrm{vs} 42.2 \pm 12 \mathrm{~mL} /$ $\left.\mathrm{cm} \mathrm{H}_{2} \mathrm{O}, P=.03\right)$ and in $\mathrm{C}_{\text {eff }}\left(32.6 \pm 9.1\right.$ vs $\left.34.8 \pm 9.4 \mathrm{~mL} / \mathrm{cm} \mathrm{H}_{2} \mathrm{O}, P=.04\right)$. During ERCC, PEF increased by $16.2 \mathrm{~L} / \mathrm{min}(P<.001)$, and flow $30 \% \mathrm{~V}_{\mathrm{T}}$ increased by $25.3 \mathrm{~L} / \mathrm{min}(P<.001)$ compared with CTRL. Six subjects $(30 \%)$ presented expiratory flow limitation (EFL) during ERCC. The effect size was small for secretion volume (0.2), $C_{s t}(0.15)$, and $C_{\text {eff }}(0.12)$ and negligible for $R_{\text {tot }}$ (0.04) and $R_{\text {init }}(0.04)$. CONCLUSIONS: Although ERCC increases expiratory flow, it has no clinically relevant effects from improving the sputum production and respiratory mechanics in hypersecretive mechanically ventilated patients. The maneuver can cause EFL in some patients. (ClinicalTrials.gov registration NCT01525121). Key words: Physical therapy modalities; intensive care; respiratory therapy; mucociliary clearance; pneumonia. [Respir Care 2014;59(5):678-685. (C) 2014 Daedalus Enterprises]
\end{abstract}

\section{Introduction}

Although a number of studies have focused on the effect of mechanical ventilation on the pulmonary parenchyma, more attention should be given to mucus clearance since its mechanisms in ventilated patients are still poorly understood. Furthermore, the advances in the understanding

The authors are affiliated with the Rehabilitation Sciences Master's Program, Augusto Motta University Center, Rio de Janeiro, Brazil, with the exception of Ms Constantino, who is affiliated with the Military Police Central Hospital, Military Police of Rio de Janeiro State, Rio de Janeiro, Brazil. Drs Guimarães and Menezes are also affiliated with the School of of simple interventions such as chest physiotherapy may contribute to reducing mucus retention and respiratory infections. ${ }^{1}$ Chest physiotherapy is an essential component of the multidisciplinary approach in critical care settings. In this context, a number of devices and manual techniques have been used to remove pulmonary secretions and re-expand collapsed areas. ${ }^{2}$ Thoracic manipulative

\footnotetext{
Physiotherapy, Federal University of Rio de Janeiro, Rio de Janeiro, Brazil. Dr Lopes is also affiliated with the Pedro Ernesto Hospital, Rio de Janeiro State University, Rio de Janeiro State, Brazil.
}

The authors have disclosed no conflicts of interest 
techniques include expiratory rib cage compression (ERCC) or "squeezing." 3 This technique aims to increase expiratory flow and stretch intercostal muscles by means of a manual thoracic compression applied during exhalation, followed by a rapid release at the onset of inspiration. ${ }^{4}$ The rationale of this technique is based on its compressive effect on the airways, increasing the air-flow velocity, which increases mucus transport. ${ }^{5}$ As the application of mechanical forces on the thorax reduces the transpulmonary pressure, rib cage compression is likely to promote pulmonary and airway collapse, diminishing respiratory compliance and/or causing expiratory flow limitation (EFL) ${ }^{6,7}$ Previous animal studies showed no beneficial effects of ERCC on respiratory mechanics and gas exchange. ${ }^{8-10}$ Moreover, Unoki et $\mathrm{al}^{9}$ postulated that the airway and alveolar collapse may be exacerbated, which agrees with the results of Martí et $a l,{ }^{10}$ who found a reduction in static compliance after applying the maneuver in an animal model. These authors also found an increase in mucus clearance with "hard expiratory rib cage compression" (brief and strong bilateral compressions); however, this was not observed in critical care patients, as reported by Unoki et al. ${ }^{4}$ Although there is no clear evidence for the physiological or airway clearance benefits of ERCC in humans, Berti et al ${ }^{11}$ found a positive effect on the duration of mechanical ventilation, as well as on the ICU discharge rate and Murray score when using ERCC along with manual hyperinflation. It is difficult to determine whether these beneficial clinical outcomes were due to ERCC, manual hyperinflation, or both, particularly because Genc et $\mathrm{al}^{12}$ observed no improvements in static compliance, gas exchange, and secretion clearance when ERCC was used with manual hyperinflation in 22 mechanically ventilated patients. It is likely that these conflicting results are due to the design differences between the studies, along with some potential confounding factors that were not considered. The present study was conducted to examine the acute mechanical effects and sputum clearance of an ERCC protocol in ventilated patients with pulmonary infection. We hypothesized that, although ERCC can promote EFL and lung collapse in some circumstances, this technique improves the airway clearance in hypersecretive mechanically ventilated patients.

Correspondence: Fernando S Guimarães PT PhD, Programa de Mestrado em Ciências da Reabilitação, Praça das Nações, 34, Bonsucesso, Rio de Janeiro CEP 21041-021, Brazil. E-mail: fguimaraes_pg@yahoo.com.br.

DOI: $10.4187 /$ respcare. 02587

\section{QUICK LOOK}

\section{Current knowledge}

Expiratory rib cage compression (ERCC) has been empirically used by respiratory therapists and physiotherapists in an attempt to enhance expiratory flows and facilitate airway clearance in mechanically ventilated patients.

\section{What this paper contributes to our knowledge}

ERCC increases expiratory flow but has no clinically relevant effects in improving the sputum production and respiratory mechanics in mechanically ventilated patients with retained secretions. ERCC can cause expiratory flow limitation in some patients.

\section{Methods}

\section{Study Design}

This was a randomized crossover study. Participants were recruited from patients admitted to an 11-bed ICU at a tertiary referral hospital. Allocation was concealed from the enrolling investigators, and randomization used 2 blocks of 10 . The study coordinator prepared sealed opaque envelopes containing a preassigned treatment order generated by computer, which were opened sequentially by the physiotherapist on the day of intervention. According to the random order, the participants received manual ERCC and the control intervention (CTRL) on the same day, with a 5-h washout period between them. Both interventions were followed by a hyperinflation maneuver using pressure support ventilation. The study protocol is depicted in Figure 1.

\section{Participants}

All subjects were receiving mechanical ventilation, presenting medical diagnoses of pulmonary infection and hypersecretion (defined as the need for suctioning $<2$-h intervals). Pulmonary infection was defined as a score of $\geq 6$, as determined by the Clinical Pulmonary Infection Score (CPIS). ${ }^{13}$ CPIS was calculated after individual scoring for each of the following parameters: (1) temperature: $36.5-38.4^{\circ} \mathrm{C}=0$ points, $38.5-38.9^{\circ} \mathrm{C}=1$ point, $\leq 36^{\circ} \mathrm{C}$ or $\geq 39^{\circ} \mathrm{C}=2$ points; (2) white blood cells $\left(\times 10^{9} / \mathrm{L}\right)$ $4.0-11.0=0$ points, $11-17=1$ point, $>17=2$ points; (3) secretions: none to minimal $=0$ points, moderate $=1$ point, large $=2$ points; (4) $\mathrm{P}_{\mathrm{aO}_{2}} / \mathrm{F}_{\mathrm{IO}_{2}}$ : $>240=0$ points, $\leq 240=2$ points; (5) chest radiograph infiltrates: clear $=0$ points, patchy $=1$ point, localized $=2$ points. Exclusion 
Mechanically ventilated patients with pulmonary infection, randomized into 2 groups 20
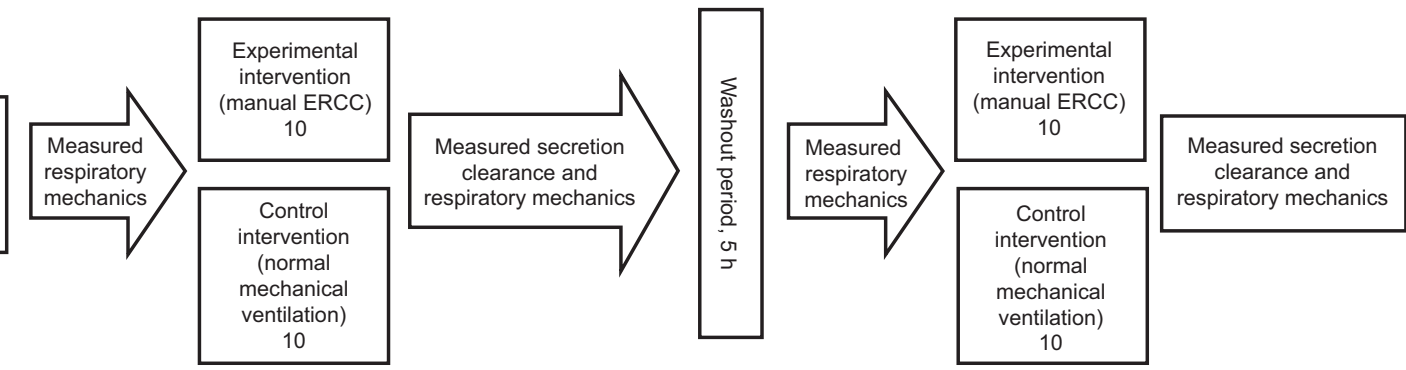

Fig. 1. Design and flow of participants through the trial. ERCC = expiratory rib cage compression.

criteria included individuals with hemodynamic instability (defined by heart rate $>130$ beats/min and/or mean arterial pressure $<60 \mathrm{~mm} \mathrm{Hg}$ ), use of vasopressor drugs, absence of respiratory drive, acute bronchospasm, ARDS, immediate postoperative neurosurgery, untreated pneumothorax, and lung hemorrhage. The research protocol was approved by the institution's ethics committee, and written informed consent was obtained from the subjects' next of kin before the study began.

\section{Intervention}

The subjects were kept in a $30^{\circ}$ semifowlers, supine position. Ventilatory mode was changed to volume-controlled mode, with a tidal volume $\left(\mathrm{V}_{\mathrm{T}}\right)$ of $8 \mathrm{~mL} / \mathrm{kg}$, an inspiratory flow of $60 \mathrm{~L} / \mathrm{min}$ (square wave), and PEEP of $5 \mathrm{~cm} \mathrm{H} \mathrm{H}_{2} \mathrm{O}\left(\mathrm{F}_{\mathrm{IO}_{2}}\right.$ remained unchanged). A first tracheal suctioning was done, and the mucus was discarded. A series of 5 min of bilateral ERCCs then ensued. Aiming to minimize intertherapist variability, the technique was applied by the same registered and trained physiotherapist. His hands were positioned on the lower ribs, and force was applied every 2 breaths only during the expiration, synchronizing the maneuver rate with the breathing frequency of the subject. The subjects then underwent a new suctioning procedure, followed by a hyperinflation maneuver consisting of a 10-min period under pressure support ventilation of $35 \mathrm{~cm} \mathrm{H}_{2} \mathrm{O}$. Control intervention followed the same sequence, but instead of the compressive maneuver, the subjects were kept on normal ventilation with the parameters described above.

\section{Outcome Measurements}

Sputum Production. Secretion clearance was the primary outcome and was measured as sputum volume $(\mathrm{mL}){ }^{14}$ Secretions were collected immediately after ERCC and CTRL, using a sputum trap attached to the suction system. Sterile saline $(10 \mathrm{~mL})$ was flushed through the suction tubing into the trap to clear any secretions in the catheter. The volume of sputum was recorded, subtracting the saline volume from the total volume in the trap. The suctioning procedure was performed following the American Association for Respiratory Care recommendations: closed suction system, suction catheter with maximal internal-toexternal diameter ratio of 0.5 , delivery of $100 \%$ oxygen $30 \mathrm{~s}$ immediately before and $1 \mathrm{~min}$ after the procedure, duration of $15 \mathrm{~s}$, and vacuum pressure of $-150 \mathrm{~mm} \mathrm{Hg.}{ }^{15}$

Respiratory Mechanics. Respiratory mechanics were recorded immediately pre-ERCC (PRE), post-ERCC or CTRL (POST1), and post-hyperinflation (POST2). There were no intervals between these protocol steps and recording of respiratory mechanics.

For the measurements, the subjects were positioned supine with the head of the bed at $30^{\circ}$ with a hyperinflated cuff and submitted to tracheal suctioning. After that, they received 3 sighs with a 2 -fold increase in $\mathrm{V}_{\mathrm{T}}$ (volume history). ${ }^{16}$ According to the end-inspiratory occlusion method, ${ }^{17}$ peak pressure $(\mathrm{P} 1)$, plateau pressure $(\mathrm{P} 2), \Delta \mathrm{P} 1$ (P1 - inflection point pressure), inspiratory flow (flow), and $\mathrm{V}_{\mathrm{T}}$ were used to calculate static $\left(\mathrm{C}_{\mathrm{st}}\right)$ and effective $\left(\mathrm{C}_{\text {eff }}\right)$ compliance of the respiratory system $\left(\mathrm{C}_{\mathrm{st}}=\mathrm{V}_{\mathrm{T}} /\right.$ $\left.[\mathrm{P} 2-\mathrm{PEEP}] ; \mathrm{C}_{\text {eff }}=\mathrm{V}_{\mathrm{T}} /[\mathrm{P} 1-\mathrm{PEEP}]\right)$ and total $\left(\mathrm{R}_{\text {tot }}\right)$ and initial $\left(\mathrm{R}_{\text {init }}\right)$ resistance of the respiratory system $\left(\mathrm{R}_{\text {tot }}=[\mathrm{P} 1-\mathrm{P} 2] /\right.$ flow; $\mathrm{R}_{\text {init }}=\Delta \mathrm{P} 1 /$ flow $)$. If there was total or partial superimposition in the expiratory flow-volume loop with and without a manual compression maneuver, this was considered EFL. ${ }^{18,19}$ In addition, peak expiratory flow (PEF) and flow at $30 \%$ of the expiratory tidal volume (flow $30 \% \mathrm{~V}_{\mathrm{T}}$; aiming to record the flow in intermediate and/or peripheral airways) were also measured during ERCC. In all steps (PRE, POST1, and POST2), the respiratory signals were collected from the ventilator display (Vela, Infrasonics, San Diego, California), and the representative value for each respiratory mechanics variable was computed as an average of 3 consistent measures.

\section{Statistical Analysis}

According to the data from Lemes et al, ${ }^{20}$ power calculation indicated that 10 participants would provide sufficient power $(80 \%)$ to detect a difference of $68 \%$ in sputum volume, assuming an SD of $67 \%$ and significance of 0.05 . 


\section{Expiratory Rib Cage Compression in Mechanically Ventilated Subjects}

Table 1. Basic Characteristics of 20 Mechanically Ventilated Subjects With Pulmonary Infection

\begin{tabular}{|c|c|}
\hline Age, mean, $\pm y$ & $65 \pm 13$ \\
\hline Median & 72 \\
\hline Interquartile range & $61-77.5$ \\
\hline \multicolumn{2}{|l|}{ Gender, $n(\%)$} \\
\hline Male & $9(45)$ \\
\hline Female & $11(55)$ \\
\hline $\mathrm{P}_{\mathrm{aO}_{2}} / \mathrm{F}_{\mathrm{IO}_{2}}$, mean $\pm \mathrm{SD}$ & $322 \pm 74$ \\
\hline APACHE II at ICU admission, mean $\pm \mathrm{SD}$ & $19 \pm 7$ \\
\hline \multicolumn{2}{|l|}{ Underlying diseases, No. $(\%)$} \\
\hline Ventilator-associated pneumonia & $13(65)$ \\
\hline Community-acquired pneumonia & $5(25)$ \\
\hline Aspirative pneumonia & $2(10)$ \\
\hline Upper abdominal surgery & $2(13.4)$ \\
\hline Congestive heart failure & $2(5)$ \\
\hline Stroke & $1(5)$ \\
\hline Septic shock & $2(10)$ \\
\hline Neurosurgery & $1(5)$ \\
\hline Kidney failure & $1(5)$ \\
\hline Pulmonary embolism & $1(5)$ \\
\hline \multicolumn{2}{|l|}{ Ventilatory mode, No. (\%) } \\
\hline VC-CMV & $4(20)$ \\
\hline PC-CMV & $4(20)$ \\
\hline PSV & $12(60)$ \\
\hline \multicolumn{2}{|l|}{ PEEP, $\mathrm{cm} \mathrm{H}_{2} \mathrm{O}$} \\
\hline Median & 5 \\
\hline Interquartile range & $5-8$ \\
\hline $\mathrm{F}_{\mathrm{IO}_{2}}$, mean \pm & $0.35 \pm 0.13$ \\
\hline Median & 0.3 \\
\hline Interquartile range & $0.3-0.38$ \\
\hline Duration of ventilation, mean $\pm \mathrm{SD} d$ & $11.2 \pm 4.5$ \\
\hline $\begin{array}{l}\text { APACHE II = Acute Physiology and Chronic Health Evaluation II } \\
\text { VC-CMV = volume controlled continuous mandatory ventilation }\end{array}$ & \\
\hline
\end{tabular}

Data were tested for normality and homogeneity of variances (Shapiro-Wilk test and Levene median test) and expressed as mean $\pm \mathrm{SD}$ (if normally distributed) or as medians in combination with quartiles and percentiles (if not normally distributed). According to data distribution, the 2-way repeated measures analysis of variance and the Tukey test were used to examine the between-interventions differences in respiratory mechanics, and the Wilcoxon test was used to compare the sputum production between CTRL and ERCC. The significance level was set at 0.05, and the SigmaStat 3.1 software (Systat Software, San Jose, California) was used for all analyses. The clinical effect of ERCC was assessed by the effect size statistic, calculated as the mean change found in a variable divided by the SD of that variable. ${ }^{21}$ We used the criteria of Cohen ${ }^{22}$ to interpret the effect size, where a value of 0.2 is considered a small effect, a value of 0.5 is considered a moderate effect, and a value of 0.8 is considered a large effect. These calculations used the POST2 data, considering CTRL and ERCC results.

\section{Results}

Data from the participants of the study are provided in Table 1 . The only center involved in this trial (Military Police of Rio de Janeiro State Hospital) has a throughput of 234 critical care patients per year, with $65 \%$ managed with mechanical ventilation. All participants completed the measurements, and their baseline respiratory mechanics values were similar before CTRL and ERCC (Table 2). One neurosurgical subject presented with a Glasgow coma score of 9. Three subjects were not sedated, and the other 16 subjects were sedated with midazolam $(0.02-0.2 \mathrm{mg} /$ $\mathrm{kg} / \mathrm{h})$ and/or fentanyl $(1.0-7.0 \mu \mathrm{g} / \mathrm{kg} / \mathrm{h})$. The infusions were titrated with the aim of maintaining levels $2-4$ in the Ramsay Sedation Scale. ${ }^{23}$ The same experienced physiotherapist delivered control and experimental interventions for all subjects, and the procedures were well tolerated without unfavorable signs and symptoms (alteration in blood pressure and/or heart rate $>$ or $<20 \%$ of resting values and desaturation of oxyhemoglobin $>10 \%$ of baseline levels). ${ }^{24}$

The respiratory mechanics and expiratory flow profiles in CTRL and ERCC are shown in Tables 2 and 3, respectively. ERCC cleared $34.4 \%$ more secretions than CTRL $(P=.04)$ (Fig. 2 and Table 2). Respiratory mechanics showed no differences between control and experimental interventions in POST1 for $\mathrm{C}_{\mathrm{st}}(P=.1), \mathrm{C}_{\mathrm{eff}}(P=.34)$, $\mathrm{R}_{\text {tot }}(P=.92)$, and $\mathrm{R}_{\text {init }}(P=.97)$. In POST2, ERCC promoted an increase in $\mathrm{C}_{\mathrm{st}}(P=.025)$ and in $\mathrm{C}_{\mathrm{eff}}$ $(P=.044)$, but not in $\mathrm{R}_{\text {tot }}(P=.92)$ and $\mathrm{R}_{\text {init }}(P=.98)$. The effect size was small for secretion volume $(0.2), \mathrm{C}_{\mathrm{st}}$ $(0.15)$, and $\mathrm{C}_{\text {eff }}(0.12)$ and negligible for $\mathrm{R}_{\text {tot }}(0.04)$ and $\mathrm{R}_{\text {init }}(0.04)$.

During ERCC, PEF increased by $16.2 \mathrm{~L} / \mathrm{min}(P<.001)$, and flow $30 \% \mathrm{~V}_{\mathrm{T}}$ increased by $25.3 \mathrm{~L} / \mathrm{min}(P<.001)$ compared with CTRL (Table 3). Six patients (30\%) presented EFL during ERCC. In a post hoc analysis comparing patients with EFL and non-EFL (NEFL), there was an increase in PEF during ERCC in NEFL $(P=.007)$, but not in $\operatorname{EFL}(P=.193)$. The flow $30 \% \mathrm{~V}_{\mathrm{T}}$ increased in EFL $(P=.043)$ and in NEFL $(P=.001)$, but NEFL presented higher values $(P=.006)$. There were correlations between $\mathrm{R}_{\text {tot }}$ and flow $30 \% \mathrm{~V}_{\mathrm{T}}$ during ERCC $(\mathrm{r}=-0.635, P=.003)$ and between $\mathrm{R}_{\text {init }}$ and flow $30 \% \mathrm{~V}_{\mathrm{T}}$ during ERCC ( $\mathrm{r}$ $=-0.596, P=.006$ ). There were no other associations between expiratory flows during ERCC and respiratory mechanics parameters. 


\section{Expiratory Rib Cage Compression in Mechanically Ventilated Subjects}

Table 2. Secretion Clearance and Respiratory Mechanics

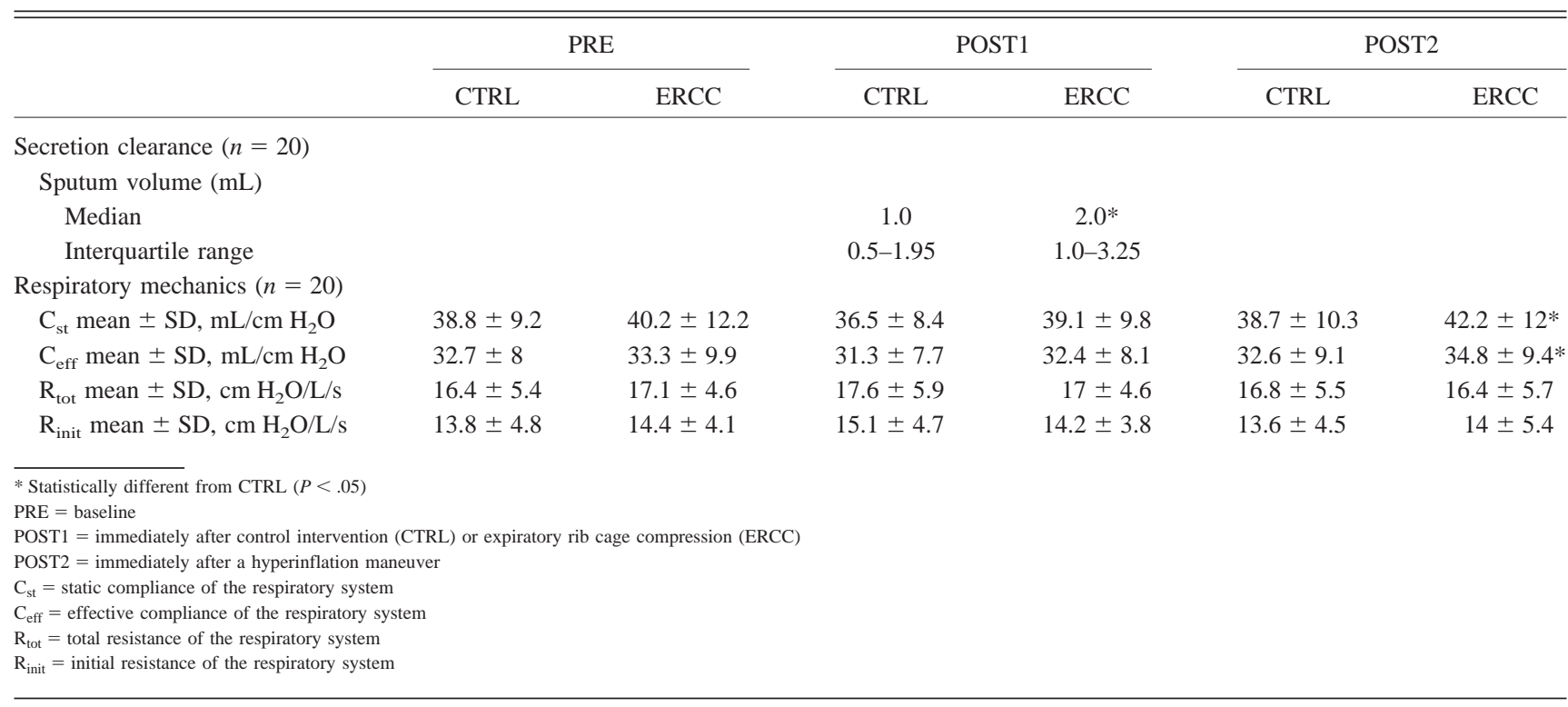

Table 3. Expiratory Flow Profiles During Expiratory Rib Cage Compression in 20 Mechanically Ventilated Subjects With Pulmonary Infection

\begin{tabular}{|c|c|c|c|c|}
\hline & \multicolumn{2}{|c|}{ PRE } & \multicolumn{2}{|c|}{ PER } \\
\hline & CTRL & ERCC & CTRL & ERCC \\
\hline $\mathrm{PEF}$ mean $\pm \mathrm{SD}, \mathrm{L} / \mathrm{min}$ & $46.4 \pm 15.2$ & $43.6 \pm 17.5$ & $46.2 \pm 15.3$ & $59.6 \pm 18.3^{*}$ \\
\hline $\begin{array}{l}\text { Flow } 30 \% \mathrm{~V}_{\mathrm{T}} \text { mean } \pm \mathrm{SD}, \mathrm{L} / \mathrm{min} \\
\text { Flow limitation, } n(\%)\end{array}$ & $15 \pm 7.3$ & $12.6 \pm 6.1$ & $15.1 \pm 7.5$ & $\begin{array}{l}38 \pm 15.7^{*} \\
6(30)\end{array}$ \\
\hline $\begin{array}{l}\text { * Statistically different from the control interv } \\
\text { PRE }=\text { baseline } \\
\text { PER }=\text { during CTRL or expiratory rib cage co } \\
\text { PEF }=\text { peak expiratory flow } \\
\text { Flow } 30 \% V_{T}=\text { flow at } 30 \% \text { of the expirator }\end{array}$ & & & & \\
\hline
\end{tabular}

\section{Discussion}

Even with lack of evidence, ERCC has been empirically used by physiotherapists for improving expiratory flows and therefore airway clearance. ${ }^{3}$ Our study showed that ERCC improves expiratory flow but has little effect on secretion removal and respiratory mechanics in mechanically ventilated patients with pulmonary infection. These results are in agreement with previous studies that found no benefits with ERCC in animal models ${ }^{8-10}$ and in mechanically ventilated patients. ${ }^{4,12}$

\section{Mucus Clearance}

In this study, mucus clearance was computed as sputum volume. Although measurement of the transport rate of mucus in the airway using a radioactive tracer technique is the most direct outcome parameter, ${ }^{5}$ this method is diffi- cult to implement in critical care settings. Thus, quantification of expectorated mucus has been used as an important airway clearance marker in most physiotherapy studies including mechanically ventilated patients. ${ }^{12,20,25-28}$ For a better mechanistic approach, our mucus clearance results are discussed along with the following respiratory mechanics subitems.

\section{Expiratory Flow Limitation}

Air-flow transport depends mainly on the air-flow velocity, which is determined by the airway diameter and the intrapulmonary pressure created by the expiratory muscles (or by any thoracic compressive maneuver). ${ }^{5}$ During a forced expiration or a compressive maneuver, the transmural pressure might reduce the airway diameter, causing a disproportion between the driving pressure and the expiratory flow (ie, EFL). If the airway collapses, the down- 


\section{Expiratory Rib Cage Compression in Mechanically Ventilated Subjects}

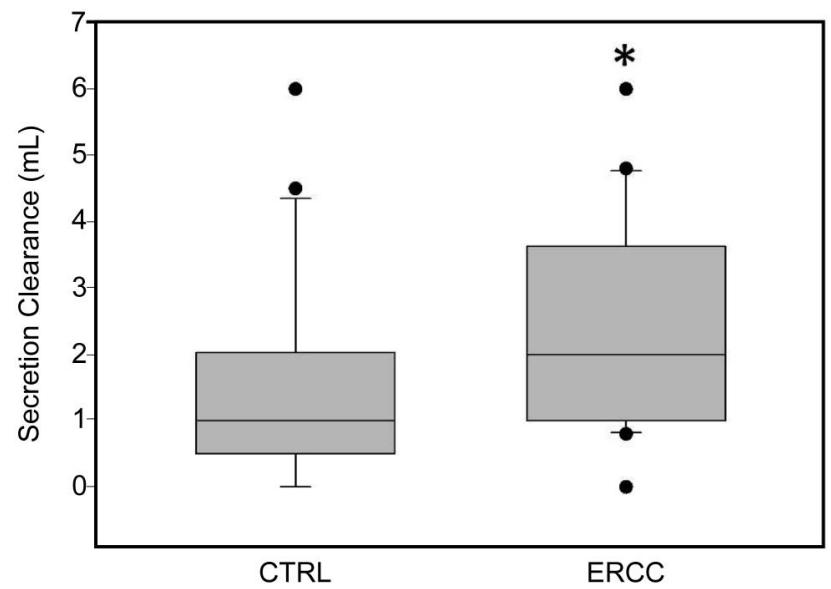

Fig. 2. Sputum volume produced after control (CTRL) and expiratory rib cage compression (ERCC) interventions. The horizontal line through each box denotes the median values, whereas the upper and lower edges represent the 25th and 75th percentiles, and the whiskers represent the 10th and 90th percentiles. Circles encompass the range of data points. The asterisk indicates significantly different from CTRL.

stream flow equals zero, and secretion removal is interrupted. ${ }^{6,29}$

Because ERCC is mechanically similar to a forced expiratory maneuver, it has the potential to cause EFL and peripheral airway closure. In our study, perhaps because all subjects were receiving mechanical ventilation with PEEP $5 \mathrm{~cm} \mathrm{H}_{2} \mathrm{O}$, only 6 subjects $(30 \%)$ presented EFL during ERCC. It is likely that the protective mechanical effect of PEEP during the maneuver prevented the EFL in many subjects and contributed to the overall increase in $\mathrm{PEF}$ and flow $30 \% \mathrm{~V}_{\mathrm{T}}$. As expected, this increase was more pronounced in NEFL subjects, suggesting that higher PEEP levels are necessary to stabilize the airway during ERCC in some patients. In this way, to increase the expiratory flow and prevent airway collapse during the maneuver, the PEEP level should be increased to the point at which there is no superposition between the baseline (current ventilation) and ERCC flow-volume loops observed on the ventilator display.

As the protective mechanical effect of PEEP is not present in spontaneous ventilated patients, it is likely that ERCC causes EFL and airway closure more frequently, mainly in patients with pulmonary obstructive diseases. However, it is impossible to predict in which patients ERCC will cause a dynamic compressive effect, which in theory can help in secretion removal, or airway collapse, which can interrupt airway clearance. On the basis of this premise, to remove peripheral secretions, the rationale of ERCC should include strategies aiming to avoid airway collapse, mainly in patients with reduced airway stability and/or functional residual capacity (eg, bed-ridden patients).

\section{Respiratory System and Airway Resistance}

We found no changes in $\mathrm{R}_{\text {tot }}$ and $\mathrm{R}_{\mathrm{init}}$, in agreement with previous work. 10,20,30,31 According to these studies, transitory bronchial constriction, variable secretion distribution patterns in patients' airways, mucociliary activity, and individualized response to the applied intervention could explain the unchanged respiratory resistance observed after the use of airway clearance techniques. Moreover, because of the greatest contribution of the central and intermediate airways to respiratory system resistance, if the secretions displace from the periphery to more proximal airways (but are not completely removed by suctioning), there will be an increase in these resistance parameters. $^{32}$

We also found that in subjects with higher respiratory and airway resistance $\left(\mathrm{R}_{\text {tot }}\right.$ and $\mathrm{R}_{\text {init }}$, respectively), the compressive maneuver was less effective in augmenting expiratory flow. This suggests that for more severely obstructed patients, a higher $\mathrm{V}_{\mathrm{T}}$ may add some benefit to ERCC in increasing expiratory flow.

\section{Static and Effective Respiratory Compliance}

Although $\mathrm{C}_{\mathrm{st}}$ has been used by several authors to assess the effect of airway clearance techniques in mechanically ventilated patients, ${ }^{20,25-28}$ the only study evaluating ERCC as an isolated technique in humans used $\mathrm{C}_{\text {eff }}{ }^{4}$ The use of this mechanical variable made the interpretation of respiratory mechanics difficult because this parameter is influenced not only by the elastic properties of the respiratory system (which depend on the recruitment or de-recruitment of lung periphery), but also by its resistive and inhomogeneous components (which depend on other factors, such as the patterns of secretion distribution in the airways). ${ }^{17}$ Nevertheless, these authors also postulated that ERCC can cause any degree of pulmonary collapse, which is in agreement with the study of Martí et al, ${ }^{10}$ who found a reduction in $\mathrm{C}_{\mathrm{st}}$ after ERCC in an animal model. In our study, $\mathrm{C}_{\mathrm{st}}$ and $\mathrm{C}_{\text {eff }}$ did not change immediately after ERCC, but presented a small increase after a hyperinflation maneuver compared with CTRL. It is likely that ERCC promoted some peripheral airway clearance in association with a degree of pulmonary collapse (because of the compressive effect), so these events counterbalanced each other, resulting in an unchanged $\mathrm{C}_{\mathrm{st}}$ in POST1. The subsequent hyperinflation maneuver re-inflated the collapsed areas, ${ }^{7}$ and the $\mathrm{C}_{\mathrm{st}}$ values then became higher in ERCC (POST2). These positive effects in respiratory mechanics after only a hyperinflation maneuver point to a new possible recommendation when using ERCC, aiming to re-inflate any collapsed pulmonary areas. However, this recommendation is applicable only if future studies show clinically relevant benefits of ERCC in some context. 


\section{Expiratory Rib Cage Compression in Mechanically Ventilated Subjects}

Our results cannot be extrapolated to other clinical settings because the patients were evaluated with a specific ventilatory mode and parameters. Indeed, the physiological and therapeutic consequences of chest physiotherapy techniques are greatly influenced by the ventilatory settings, as, for example, the role of PEEP in preventing airway and lung collapse. Additionally, because there are no favorable data on ERCC short-term effects in the current literature, we cannot expect a positive impact of this technique in clinically relevant outcomes, such as stay, weaning outcome, mortality, and incidence of ventilatorassociated pneumonia. The only study on these outcomes applied ERCC along with manual hyperinflation, making it difficult to determine which of these two techniques was responsible for the improvement in the stay and time-toweaning found by the authors. ${ }^{11}$

Regarding the limitations of the study, arterial blood gases were not recorded, and a blinded assessor could have done all measurements. Despite these limitations, we believe that our results add an important contribution to the field because we assessed the isolated clinical and physiological effects of ERCC (not associated with any other technique), which confirmed the negative results from previous animal and human studies. Moreover, in our study, we took special care to ensure that sputum production and respiratory mechanics reflected the effects of ERCC on lung periphery. In this way, before the interventions, all subjects were suctioned to remove the secretions from central airways, and measurements of respiratory mechanics included $\mathrm{C}_{\mathrm{st}}$, a recognized marker of peripheral airway clearance. ${ }^{33} \mathrm{We}$ also suggested possible recommendations for this technique, such as the use of a hyperinflation maneuver after its application and flow-volume curve monitoring during thoracic compression to avoid airway collapse by setting an appropriate PEEP level.

Additional studies are necessary to evaluate the effects of ERCC in spontaneous ventilation, in specific diseases and respiratory mechanics conditions, and with different ventilatory modes and parameters. It is likely that other factors, such as compressive force magnitude, airway stability, and pulmonary volume at the onset of expiration, influence the effectiveness of ERCC. Additionally, for a better understanding of the underlying mechanisms of ERCC, it is important to consider not only the PEF, but also fluid mechanics principles, which determine mucus transport by air-liquid interaction in central and peripheral airways.

In conclusion, although ERCC increases expiratory flow, it has no clinically relevant effects in improving the sputum production and respiratory mechanics in hypersecretive mechanically ventilated patients. The maneuver can cause EFL in some patients.

\section{REFERENCES}

1. Siner JM. An exogenous cough. Crit Care Med 2013;41(3):929-930.

2. Gosselink R, Bott J, Johnson M, Dean E, Nava S, Norrenberg M, et al. Physiotherapy for adult patients with critical illness: recommendations of the European Respiratory Society and European Society of Intensive Care Medicine Task Force on Physiotherapy for Critically Ill Patients. Intensive Care Med 2008;34(7):1188-1199.

3. Branson RD. Secretion management in the mechanically ventilated patient. Respir Care 2007;52(10):1328-1342, discussion 13421347

4. Unoki T, Kawasaki Y, Mizutani T, Fujino Y, Yanagisawa Y, Ishimatsu $\mathrm{S}$, et al. Effects of expiratory rib-cage compression on oxygenation, ventilation, and airway-secretion removal in patients receiving mechanical ventilation. Respir Care 2005;50(11):14301437.

5. Van der Schans CP. Bronchial mucus transport. Respir Care 2007; 52(9):1150-1156, discussion 1156-1158.

6. Selsby D, Jones JG. Some physiological and clinical aspects of chest physiotherapy. Br J Anaesth 1990;64(5):621-631.

7. Guimarães FS, Zin WA. Thoracic percussion yields reversible mechanical changes in healthy subjects. Eur J Appl Physiol 2008;104(4): 601-607.

8. Unoki T, Mizutani T, Toyooka H. Effects of expiratory rib cage compression and/or prone position on oxygenation and ventilation in mechanically ventilated rabbits with induced atelectasis. Respir Care 2003;48(8):754-762.

9. Unoki T, Mizutani T, Toyooka H. Effects of expiratory rib cage compression combined with endotracheal suctioning on gas exchange in mechanically ventilated rabbits with induced atelectasis. Respir Care 2004;49(8):896-901.

10. Martí JD, Li Bassi G, Rigol M, Saucedo L, Ranzani OT, Esperatti M, et al. Effects of manual rib cage compressions on expiratory flow and mucus clearance during mechanical ventilation. Crit Care Med 2013; 41(3):850-856.

11. Berti JS, Tonon E, Ronchi CF, Berti HW, Stefano LM, Gut AL, et al. Manual hyperinflation combined with expiratory rib cage compression for reduction of length of ICU stay in critically ill patients on mechanical ventilation. J Bras Pneumol 2012;38(4):477-486.

12. Genc A, Akan M, Gunerli A. The effects of manual hyperinflation with or without rib-cage compression in mechanically ventilated patients. Ital J Physiother 2011;1(2):48-54.

13. Singh N, Rogers P, Atwood CW, Wagener MM, Yu VL. Shortcourse empiric antibiotic therapy for patients with pulmonary infiltrates in the intensive care unit. A proposed solution for indiscriminate antibiotic prescription. Am J Respir Crit Care Med 2000;162(2): 505-511.

14. van der Schans CP. Airway clearance: assessment of techniques. Paediatr Respir Rev 2002;3(2):110-114.

15. American Association for Respiratory Care. AARC Clinical Practice Guidelines. Endotracheal suctioning of mechanically ventilated patients with artificial airways 2010. Respir Care 2010;55(6):758764.

16. Mead J, Collier C. Relation of volume history of lungs to respiratory mechanics in anesthetized dogs. J Appl Physiol 1959;14(5):669-678.

17. Bates JH, Rossi A, Milic-Emili J. Analysis of the behavior of the respiratory system with constant inspiratory flow. J Appl Physiol 1985;58(6): 1840-1848

18. Koutsoukou A, Armaganidis A, Stavrakaki-Kallergi C, Vassilakopoulos T, Lymberis A, Roussos C, Milic-Emili J. Expiratory flow limitation and intrinsic positive end-expiratory pressure at zero positive end-expiratory pressure in patients with adult respiratory distress syndrome. Am J Respir Crit Care Med 2000;161(5):1590-1596. 


\section{Expiratory Rib Cage Compression in Mechanically Ventilated Subjects}

19. Calverley PM, Koulouris NG. Flow limitation and dynamic hyperinflation: key concepts in modern respiratory physiology. Eur Respir J 2005;25(1):186-199.

20. Lemes DA, Zin WA, Guimarães FS. Hyperinflation using pressure support ventilation improves secretion clearance and respiratory mechanics in ventilated patients with pulmonary infection: a randomised crossover trial. Aust J Physiother 2009;55(4):249-254.

21. Kazis LE, Anderson JJ, Meenan RF. Effect sizes for interpreting changes in health status. Med Care 1989;27(3 Suppl):S178-S189.

22. Cohen J. Statistical power analysis for the behavioral sciences. London: Academic Press; 1977:1-20.

23. Ramsay MA, Savege TM, Simpson BR, Goodwin R. Controlled sedation with alphaxalone-alphadolone. Br Med J 1974;2(5920):656-659.

24. Zeppos L, Patman S, Berney S, Adsett JA, Bridson JM, Paratz JD. Physiotherapy in intensive care is safe: an observational study. Aust J Physiother 2007;53(4):279-283.

25. Berney S, Denehy L. A comparison of the effects of manual and ventilator hyperinflation on static lung compliance and sputum production in intubated and ventilated intensive care patients. Physiother Res Int 2002;7(2):100-108.

26. Savian C, Paratz J, Davies A. Comparison of the effectiveness of manual and ventilator hyperinflation at different levels of positive end-expiratory pressure in artificially ventilated and intubated intensive care patients. Heart Lung 2006;35(5):334-341.

27. Chicayban LM, Zin WA, Guimarães FS. Can the Flutter Valve improve respiratory mechanics and sputum production in mechanically ventilated patients? A randomized crossover trial. Heart Lung 2011; 40(6):545-553.

28. Dennis D, Jacob W, Budgeon C. Ventilator versus manual hyperinflation in clearing sputum in ventilated intensive care unit patients. Anaesth Intensive Care 2012;40(1):142-149.

29. Oberwaldner B. Physiotherapy for airway clearance in paediatrics. Eur Respir J 2000;15(1):196-204.

30. Guglielminotti J, Desmonts JM, Dureuil B. Effects of tracheal suctioning on respiratory resistances in mechanically ventilated patients. Chest 1998;113(5):1335-1338.

31. Mackenzie CF, Shin B. Cardiorespiratory function before and after chest physiotherapy in mechanically ventilated patients with posttraumatic respiratory failure. Crit Care Med 1985;13(6):483-486.

32. Weibel ER. Morphometry of the human lung. New York: Academic Press; 1963:84-85

33. Winning TJ, Brock-Utne JG, Goodwin NM. A simple clinical method of quantitating the effects of chest physiotherapy in mechanically ventilated patients. Anaesth Intensive Care 1975;3(3):237-238. 